\title{
PARTON INTRINSIC MOTION: UNPOLARIZED CROSS SECTIONS AND THE SIVERS EFFECT IN INCLUSIVE PARTICLE PRODUCTION*
}

\author{
U. D'ALESIO AND F. MURGIA \\ Dipartimento di Fisica, Università di Cagliari and \\ INFN, Sezione di Cagliari, C.P. 170, 09042 Monserrato (CA), Italy
}

\begin{abstract}
We present a detailed study, performed in the framework of LO perturbative QCD with the inclusion of spin and $\boldsymbol{k}_{\perp}$ effects, of unpolarized cross sections for the Drell-Yan process and for inclusive $\pi$ and $\gamma$ production in hadronic collisions, in different kinematical situations. We find a satisfactory agreement between theoretical predictions and experimental data. This supports the study of spin effects and transverse single spin asymmetries (SSA) within the same scheme. We then present results for SSA, generated by the so-called Sivers effect, in inclusive pion production in proton-proton collisions.
\end{abstract}

It is known that collinear pQCD, even at NLO, often underestimates experimental results for inclusive pion and photon production in hadronic collisions in the central rapidity region and at moderately large $p_{T}$. It has also been shown that intrinsic parton momentum $\left(\boldsymbol{k}_{\perp}\right)$ effects reconcile in most cases theoretical calculations with experimental results. The role of $\boldsymbol{k}_{\perp}$ effects has been also studied in the context of polarized highenergy inclusive particle production at moderately large $p_{T}$, in particular at medium-large $x_{F}$. Two noticeable examples are the SSA observed in $p^{\uparrow} p \rightarrow \pi X$ processes, and the transverse $\Lambda$ polarization measured in unpolarized hadronic collisions. It was originally suggested by Sivers ${ }^{1}$ and Collins $^{2}$ that pQCD with proper inclusion of spin and $\boldsymbol{k}_{\perp}$ effects in parton distribution/fragmentation functions $(\mathrm{PDF} / \mathrm{FF})$ and in elementary dynamics could be able to explain experimental results on $A_{N}(\pi)$. This suggestion has been further extended in a number of subsequent papers. ${ }^{3}$ Recently, however, Bourrely and Soffer ${ }^{4}$ have claimed that most of the experimental data on SSA cannot be explained within PQCD, on the basis that collinear pQCD fails to reproduce the corresponding unpolarized cross sections by 1-2 orders of magnitude. As a matter of fact, in early pQCD-based approaches to SSA a careful analysis of unpolarized cross sections was not addressed. Therefore, it was timely to perform a detailed and comprehensive study of unpolarized cross sections and SSA within the same pQCD

*Talk delivered by F. Murgia at the "16th International Spin Physics Symposium", SPIN2004, October 10-16, 2004, Trieste, Italy. 
approach and at the same level of accuracy. In this contribution we summarize the main results of this program. A detailed presentation and a complete list of references can be found in Ref. 5 .

In order to study the inclusive production of moderately large $p_{T}$ particles in high-energy hadron collisions, the process $A B \rightarrow C X$, we generalize, by including spin and $\boldsymbol{k}_{\perp}$ effects, the well-known collinear pQCD factorized expression for the corresponding differential cross section:

$$
\begin{aligned}
& \frac{E_{C} d \sigma^{A B \rightarrow C X}}{d^{3} \boldsymbol{p}_{C}}=\sum_{a, b, c, d} \int d x_{a} d x_{b} d z \prod_{i} d^{2} \boldsymbol{k}_{\perp i} \hat{f}_{a / A}\left(x_{a}, \boldsymbol{k}_{\perp a}\right) \hat{f}_{b / B}\left(x_{b}, \boldsymbol{k}_{\perp b}\right) \\
& \times \frac{\hat{s}}{x_{a} x_{b} s} \frac{d \hat{\sigma}^{a b \rightarrow c d}}{d \hat{t}}\left(x_{a}, x_{b}, \hat{s}, \hat{t}, \hat{u}\right) \frac{\hat{s}}{\pi} \delta(\hat{s}+\hat{t}+\hat{u}) \frac{1}{z^{2}} J\left(z,\left|\boldsymbol{k}_{\perp C}\right|\right) \hat{D}_{C / c}\left(z, \boldsymbol{k}_{\perp C}\right) .
\end{aligned}
$$

The factors $\hat{s} /\left(x_{a} x_{b} s\right)$ and $J\left(z,\left|\boldsymbol{k}_{\perp C}\right|\right)$ are due to $\boldsymbol{k}_{\perp}$ effects on the kinematics and become unity in collinear pQCD with massless partons. The rest of the notation should be obvious. ${ }^{5}$ Additional contributions to the unpolarized cross section are in principle possible; however, it can be shown that they are negligible. ${ }^{5,6}$ When considering SSA and Sivers effect only, to get the numerator of the asymmetry (the denominator being twice the unpolarized cross section) one simply substitutes in this master formula the unpolarized PDF with the corresponding Sivers function for the parton inside the initial polarized hadron. Notice that a formal proof of factorization with the inclusion of $\boldsymbol{k}_{\perp}$ effects is still missing for $A B \rightarrow C X$ processes. Moreover, a complete formal definition of spin and $\boldsymbol{k}_{\perp}$ dependent $\mathrm{PDF} / \mathrm{FF}$, including their evolution and universality properties is also missing; we also lack a consistent higher-twist treatment including additional, unknown (higher-twist) PDF/FF and possible quark-gluon correlations. In fact, only "enhanced" higher-twist effects due to $\boldsymbol{k}_{\perp}$ 's are included in our approach: i) the change of the $x_{a, b}, z$ regions contributing mostly to the integrals in our master formula can have a substantial effect, particularly at large $x / z$ values, where $\mathrm{PDF} / \mathrm{FF}$ vary rapidly; ii) the partonic scattering angle in the $A B$ c.m. frame might become much smaller than the hadronic production angle, thus enhancing the moderately large $p_{T}$ production of particles.

Let us now summarize some basic details of numerical calculations: a) We use a factorized, Gaussian-like and flavour-independent behaviour for the $\boldsymbol{k}_{\perp}$-dependent part of $\mathrm{PDF} / \mathrm{FF}$, e.g. $\hat{f}_{a / A}\left(x_{a}, \boldsymbol{k}_{\perp a}\right)=$ $f_{a / A}\left(x_{a}\right)\left(\beta^{2} / \pi\right) \exp \left[-\beta^{2} k_{\perp a}^{2}\right]$ (where $1 / \beta=\left\langle k_{\perp a}^{2}\right\rangle^{1 / 2}$ ); $b$ ) We calculate partonic cross sections at LO in $\alpha_{s}$, with $\boldsymbol{k}_{\perp}$-modified partonic invariants $\hat{s}, \hat{t}, \hat{u}$, introducing a regulator mass, $\mu=0.8 \mathrm{GeV}$, so that 
$\left.\hat{t} \rightarrow \hat{t}-\mu^{2}, \hat{u} \rightarrow \hat{u}-\mu^{2}, \hat{s} \rightarrow \hat{s}+2 \mu^{2} ; c\right)$ We use a unique scale for renormalization and factorization, $Q=\hat{p}_{T}^{*} / 2, \hat{p}_{T}^{*}$ being the transverse momentum of the fragmenting parton in the partonic c.m frame; $d$ ) We properly take into account NLO corrections ( $K$-factors); our LO numerical results are always rescaled in the plots by a fixed (for a given curve) $K$-factor, estimated by using the numerical code INCNLL. ${ }^{7} e$ ) We adopt MRST01 PDF, and Kretzer/KKP pion FF; $f$ ) No complete best-fit procedure has been attempted at this stage; therefore, in principle our results may be further improved.
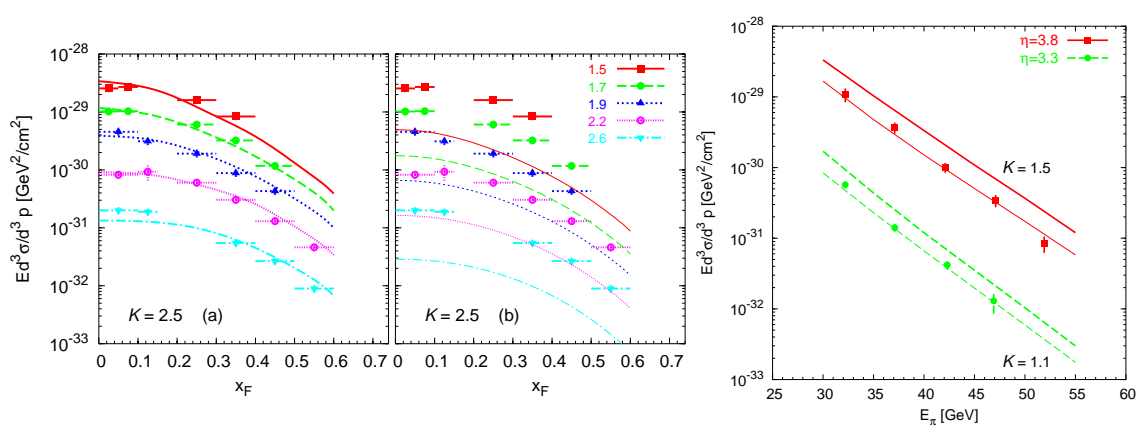

Figure 1. Cross section for $p p \rightarrow \pi X$ process, with (thick lines) and without (thin lines) $\boldsymbol{k}_{\perp}$ effects, compared to $\mathrm{FNAL}^{8}$ (left) and $\mathrm{STAR}^{9}$ (right) data.

The following processes and kinematical configurations have been considered: 1) $p p \rightarrow \mu^{+} \mu^{-} X$, in the region $20 \leq \sqrt{s} \leq 60 \mathrm{GeV}, 5 \leq M \leq 10$ $\mathrm{GeV}, q_{T}<3 \mathrm{GeV} / c$ [used also to estimate $1 / \beta=\left\langle k_{\perp a, b}^{2}\right\rangle^{1 / 2}=0.8 \mathrm{GeV} / c$ for $\mathrm{PDF}]$; 2) $p p \rightarrow \gamma X, \bar{p} p \rightarrow \gamma X$, in the region $20 \leq \sqrt{s} \leq 60 \mathrm{GeV}$, $1.5 \leq p_{T} \leq 10 \mathrm{GeV} / c,\left|x_{F}\right|<0.4$, and $\sqrt{s} \simeq 600 \mathrm{GeV}, 10 \leq p_{T} \leq 80$ $\mathrm{GeV} / c$; 3) $p p \rightarrow \pi X$ in the region $20 \leq \sqrt{s} \leq 200 \mathrm{GeV}, 1.5 \leq p_{T} \leq 14$ $\mathrm{GeV} / c,\left|x_{F}\right|<0.8$ [used to estimate $1 / \beta^{\prime}=\left\langle k_{\perp \pi}^{2}\right\rangle^{1 / 2}$, see Ref. 5]. We can only present few representative results here: in Fig. 1 we show the differential unpolarized cross section for $p p \rightarrow \pi X$ in the kinematical configurations of Ref. 8 (left) and of the STAR experiment at RHIC (right); this gives a comparison between a calculation similar to that of Ref. 4 (thin lines) and our results including $\boldsymbol{k}_{\perp}$ effects (thick lines). In Fig. 2, we show $A_{N}\left(p^{\uparrow} p \rightarrow \pi X\right)$ in the kinematical configurations of the E704 experiment at FNAL (left) [used to fix the Sivers function] and of the STAR experiment at BNL (right).

In conclusion, our LO approach (complemented with proper NLO $K$ factors) is in reasonable agreement with a large set of experimental data for unpolarized cross sections. This gives support to the validity of the same 

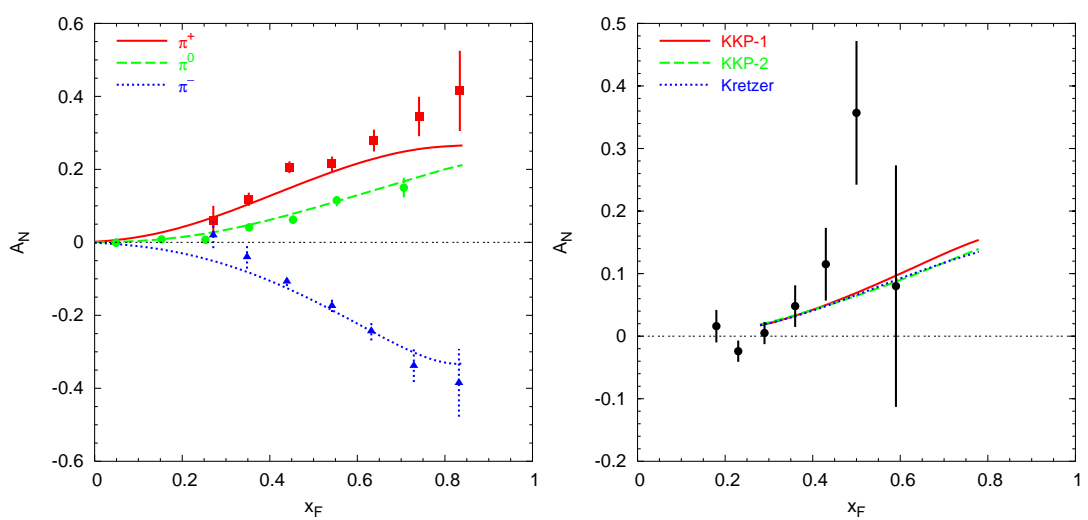

Figure 2. $\quad A_{N}\left(p^{\uparrow} p \rightarrow \pi X\right)$ compared to E704 ${ }^{10}$ (left) and $\mathrm{STAR}^{9}$ (right) data.

approach in the study of SSA. A detailed treatment of $\boldsymbol{k}_{\perp}$ effects confirms all main results and conclusions of our former studies on Sivers effect performed keeping only leading contributions (in $\boldsymbol{k}_{\perp}$ ) and using a simplified partonic configuration (see the first of Ref. 3). Let us finally notice that our approach is in principle able to reproduce most of the features of the experimental data available for $P_{T}^{\Lambda}\left(p p \rightarrow \Lambda^{\uparrow} X\right)$. A combined analysis of $P_{T}^{\Lambda}$ and unpolarized cross sections, like that performed here for the Drell-Yan process and SSA in inclusive $\pi$ and $\gamma$ production, is under way.

We acknowledge partial support from "Cofinanziamento MIURPRIN03".

\section{References}

1. D. Sivers, Phys. Rev. D41, 83 (1990); Phys. Rev. D43, 261 (1991)

2. J.C. Collins, Nucl. Phys. B396, 16 (1993)

3. See, e.g., M. Anselmino, F. Murgia, Phys. Lett. B442, 470 (1998); M. Anselmino, M. Boglione, F. Murgia, Phys. Rev. D60, 054027 (1999)

4. C. Bourrely, J. Soffer, Eur. Phys. J. C36, 371 (2004)

5. U. D'Alesio and F. Murgia, Phys. Rev. D70 074009 (2004)

6. M. Anselmino, M. Boglione, U. D'Alesio, E. Leader, F. Murgia, e-Print Archive: hep-ph/0408356 (Phys. Rev. D, in press)

7. P. Aurenche, et al., Nucl. Phys. B297, 661 (1988)

8. G. Donaldson, et al., Phys. Lett. B73, 375 (1978)

9. STAR Collaboration, J. Adams, et al., Phys. Rev. Lett. 92, 171801 (2004); S. Heppelmann, contribution to the Transversity Workshop, October 6-7, 2003, IASA, Athens, Greece

10. E704 Collaboration, D.L. Adams et al., Phys. Lett. B345, 569 (1995); 261, 201 (1991); 264, 462 (1991) 